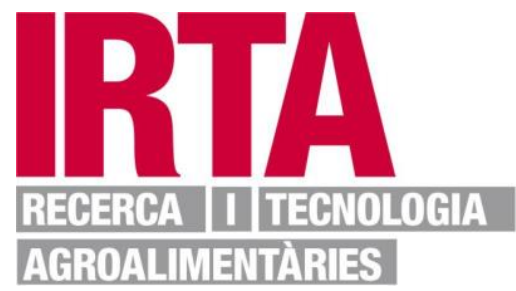

This document is a postprint version of an article published in Meat Science () Elsevier after peer review. To access the final edited and published work see https://doi.org/10.1016/j.meatsci.2018.04.011 


\title{
1 On-line Ham Grading using pattern recognition models based 2 on available data in commercial pig slaughterhouses
}

3 Gerard Masferrer $^{\mathrm{ab}}$, Ricard Carreras ${ }^{\mathrm{b}}$, Maria Font-i-Furnols ${ }^{\mathrm{c}}$, Marina Gispert ${ }^{\mathrm{c}}$, Pere Marti-Puig $^{\mathrm{a}}$, Moises Serra ${ }^{\mathrm{a}}$

4

5

6

7

8

anformation and Digital Technology Department, UVic-UCC, Vic (Barcelona)

${ }^{b}$ Mafrica.SA, Paratge Can Canals Nou, S/N 08250 Sant Joan de Vilatorrada (BCN)

'IRTA-Product Quality, 17121 Monells, Girona, Spain

\begin{abstract}
The thickness of the subcutaneous fat in hams is one of the most important factors for the drycuring process and largely determines its final quality. This parameter is usually measured in slaughterhouses by a manual metrical measure to classify hams. The aim of the present study was to propose an automatic classification method based on data obtained from a carcass automatic classification equipment (AutoFom) and intrinsic data of the pigs (sex, breed, and weight) to simulate the manual classification system. The evaluated classification algorithms were decision tree, support vector machines (SVM), k-nearest neighbour and discriminant analysis. A total of 4000 hams selected by breed and sex were classified as thin (0-10mm), standard (11-15 mm), semi-fat $(16-20 \mathrm{~mm})$ and fat $(>20 \mathrm{~mm})$. The most reliable model, with a percentage of success of $73 \%$, was SVM with Gaussian kernel, including all data available. These results suggest that the proposed classification method can be a useful online tool in slaughterhouses to classify hams.
\end{abstract}

Keywords dry-cured hams; ham-fat grading; subcutaneous fat thickness; pattern recognition

\section{Introduction}

Ham is one of the most valued product in pork meat industry. This primal cut represents between 25 and 30 percent of the carcass (Cisneros, Ellis, \& McKeith, 1996; Gispert et al., 2007) and is the basis of different regional specialities focused on preserving and flavouring raw meat (Dirinck, Van Opstaele, \& Vandendriessche, 1997). Those specialities include different techniques such as salting dry-cured ham, smoking or wet curing. Some examples are Westphalian ham in Germany, Prosciutto in Italy, and Jamon Serrano in Spain.

The Subcutaneous Fat Thickness (SFT) in hams determines, among other factors, which is the best process for the ham to be submitted. Hams with low subcutaneous fat have a high lean meat percentage (LMP) and are more appropriate to be processed as raw or cooked meat while hams with higher subcutaneous fat are more appropriate to be cured or smoked. 
Moreover, the SFT determines the optimum curing time (Bosi, Russo, \& Paolo, 2004), which is directly related to the quality of the final product (Čandek-Potokar \& Škrlep, 2012). Therefore, classify the ham according to the SFT is crucial to get the maximum benefit of the product, in economic and quality terms.

The thickness of the subcutaneous fat is determined by several factors, among which can be highlighted the breed (Gispert et al., 2007; Wood et al., 2004), the sex (Font-i-Furnols et al., 2012; Gispert et al., 2010), the slaughter weight (Fàbrega et al., 2011; Latorre, García-Belenguer, \& Ariño, 2008) and the diet (Realini et al., 2010; Tous et al., 2014; Wood et al., 2004). Regarding the breed, there are leaner breeds, as would be the Pietrain and other fattier breeds such as the Duroc (Cilla et al., 2006; Edwards, Bates, \& Osburn, 2003). In terms of sex, females tend to deposit more subcutaneous fat than males (Gispert et al., 2010; Wood, Enser, Whittington, Moncrieff, \& Kempster, 1989). Moreover, the castration, especially surgical but also immunological, also contributes to deposit more subcutaneous-fat compared with entire male pigs (Gispert et al., 2010; Wood et al., 2008).

Nowadays slaughterhouses have different methods to estimate the SFT of hams. One of the most used method is the visual system based on a metrical measure of the SFT over the Gluteus medius muscle, similar to ZP (Zwei-Punkte Messverfahren) measures, used to determine carcass LMP (Daumas, 2011; Font-i-Furnols et al., 2016). Indeed, the carcass LMP is a parameter widely used in slaughterhouses as the current EU legislation establishes it as compulsory for carcasses classification. There are different methods to determine LMP based, predominantly, on the existing relationship of thickness between fat and muscle in several parts of the carcass (Font $\mathrm{i}$ Furnols \& Gispert, 2009).

Obtaining these measures manually is unsuitable in slaughter plants with medium/high speed line, therefore the most used methods to determine LMP are semiautomatic systems based on reflectance penetration probes, as for instance the Fat-O-Meat'er (FOM; Frontmatec Smørum A/S, Herlev, Denmark) or the Hennessy Grading Probe (HGP; Hennessy Grading System Ltd., Auckland, New Zealand), which determine fat and muscle thickness at a defined anatomical position and use them to estimate carcass LMP. Alternatively, there are non-invasive and fully automatic systems such as AutoFom (Frontmatec Smørum A/S, Herlev, Denmark) which is based on three-dimensional ultrasonic systems, or VCS 2000 (e + V Technology GmbH, Oranienburg, Germany) that extracts LMP by processing and analysing images (Font i Furnols \& Gispert, 2009). Some of these devices also can estimate several SFT at the loin and at the ham level. For instance, AutoFom, provides several SFT parameters of the ham like fatham2 (minimum subcutaneous fat plus skin thickness measured with a ruler over the muscle Gluteus medius) and fatham3 (thickness of the subcutaneous fat plus skin measured with a ruler, perpendicularly to the 
skin, at the cranial part of muscle Gluteus medius).

Other systems, such as thermography technology have been proposed to classify the hams according to the SFT, being the hams with lower fat cover the ones that display a significantly warmer average temperature surface (Nanni Costa et al., 2010). Also computed tomography has been used in experimental conditions to determine the fat thickness at different anatomical positions mainly in the loin region (Lucas et al., 2017) although it could also been used in the ham region as has been done in live pigs (Carabús et al., 2014).

Nowadays a certain amount of data is collected in the slaughter line like gender and carcass weight, but also much other information from the productive chain is available such as breed, diet, transport and farm conditions, medication and castration (if done). In this context, with all this available data it is possible to take technical and commercial real-time decisions to better classify products and maximise profits. Therefore, our hypothesis is that complementing the Autofom-III set of estimated parameters with those additional ones could be used to improve the ham classification rate according to the SFT.

To carry out this classification it is possible to use classifiers. A classifier is an algorithm used to assign an unlabelled incoming element in a known category based on certain characteristic information of that element. These algorithms need to perform a learning stage. There are two types of primary learning strategies: supervised learning which elaborates a mathematical function (hypothesis) from previously labelled training data and unsupervised learning which does not have a training package that allows knowing the data labels, so it is necessary to use grouping techniques that try to build these labels. Among supervised algorithms, some of the most widespread are Decision Trees, K-Nearest Neighbours $(K N N)$, Linear and Nonlinear Discriminant Analysis (LDA/nLDA) and Support Vector Machine (SVM) (Bishop, 2006). Between the unsupervised classifiers the most popular strategies are the clustering which includes the Hierarchical and k-Means clustering algorithms.

Thus, the objectives of this study are: (1) To apply and assess different supervised classification techniques (Decision trees, $\mathrm{kNN}, \mathrm{SVN}, \mathrm{LDA} / \mathrm{nLDA}$ ) to predict the classification of hams according to SFT by combining data form Autofom III and intrinsic data from the animal, (2) to evaluate the impact of each predictor in the accuracy of ham classification, and (3) to evaluate several combinations of predictors available in different slaughterhouses scenarios and to compare them.

\section{Material and Methods}


102 This study was carried out with data obtained during May 2016 from pigs fattened in Spanish 103 commercial farms and slaughtered in a commercial slaughterhouse (MAFRICA S.A.) located in 104 Sant Joan de Vilatorrada, Catalonia, Spain. All farms were less than $200 \mathrm{~km}$ far from the 105 slaughterhouse and pigs were transported using trucks in groups (usually of between 80 and 220 106 animals). Once in the slaughterhouse pigs rested into lairage pens between 2 and 4 hours before 107 being slaughtered.

108 This slaughterhouse works five days per week slaughtering a mean of 1700 pigs per day, obtaining 109 more than 32000 carcasses per month. A total of 4000 carcasses were selected for this study 110 according to their breed and sex in order to ensure a representative sample regarding fat thickness.

111 Those carcasses were selected according to their sex: $60.6 \%$ females, $19.4 \%$ entire males and $11220.0 \%$ castrated males and according to their genetics: 51.9\% (Large White $\times$ Landrace) $\times$ 113 Piétrain, 38.3\% were (Large White $\mathrm{x}$ Landrace) $\mathrm{x}$ Duroc and 9.8\% (Large White $\mathrm{x}$ Landrace) $\mathrm{x}$ 114 (Duroc $\mathrm{x}$ Landrace). Table 1 shows the mean weight of the cold carcass and the fat thickness of 115 the pigs according to the breed and sex. Fat thickness parameter is given by the ultrasound 116 AutoFom-III system and corresponds to the parameter F34 that is described as the fat thickness 117 at $60 \mathrm{~mm}$ in the mid-line between the $3 \mathrm{rd}$ and the 4th last rib.

118 Table 1. The cold carcass weight (mean $\pm \mathrm{s.d} ; \mathrm{kg}$ ) and the fat thickness at $60 \mathrm{~mm}$ in the mid-line between the $3^{\text {rd }}$ and 119 the $4^{\text {th }}$ last rib (mean \pm s.d.; $\mathrm{mm}$ ) of 4000 carcass according to breed and sex.

\begin{tabular}{llll}
\hline BREED & $\mathbf{n}$ & $\begin{array}{l}\text { WEIGHT } \\
(\mathbf{m e a n} \pm \mathbf{s . d} \text {; } \mathbf{~ g g})\end{array}$ & $\begin{array}{l}\text { FAT THICKNESS } \\
(\mathbf{m e a n} \pm \text { s.d.; mm) }\end{array}$ \\
\hline (Large White $\times$ Landrace) $\times$ Piétrain & 2077 & $81.80 \pm 8.16$ & $15.39 \pm 4.10$ \\
(Large White x Landrace) x Duroc & 1531 & $93.76 \pm 10.69$ & $24.55 \pm 5.56$ \\
$\begin{array}{l}\text { (Large White x Landrace) x (Duroc x } \\
\text { Landrace) }\end{array}$ & 392 & $85.92 \pm 9.02$ & $18.60 \pm 5.20$ \\
\hline SEX & & & \\
\hline Female & 2289 & $85.49 \pm 10.00$ & $17.59 \pm 5.63$ \\
Castrated & 1315 & $90.97 \pm 11.54$ & $23.51 \pm 6.19$ \\
Entire male & 396 & $80.38 \pm 7.91$ & $14.29 \pm 3.22$ \\
\hline
\end{tabular}

121 Pigs were slaughtered after stunning with $\mathrm{CO}_{2}(90 \%)$ for $2 \mathrm{~min}$. After scalding they were totally 122 monitored using the ultrasound AutoFom-III system. Then pigs were eviscerated and splitted 123 according to standard commercial procedures using an automatic robotic system. After that, the 124 two half-carcasses were weighted and an experimented operator visually determined the sex of 125 the pig (female, entire male or castrated male) and classified the left half carcass according to 126 minimal fat depth over muscle gluteus medius which is shown in Fig. 1. Classes were established 127 based on the measures shown in Table 2. The operator had a pattern, based on these classes, that 

classified.

130

135

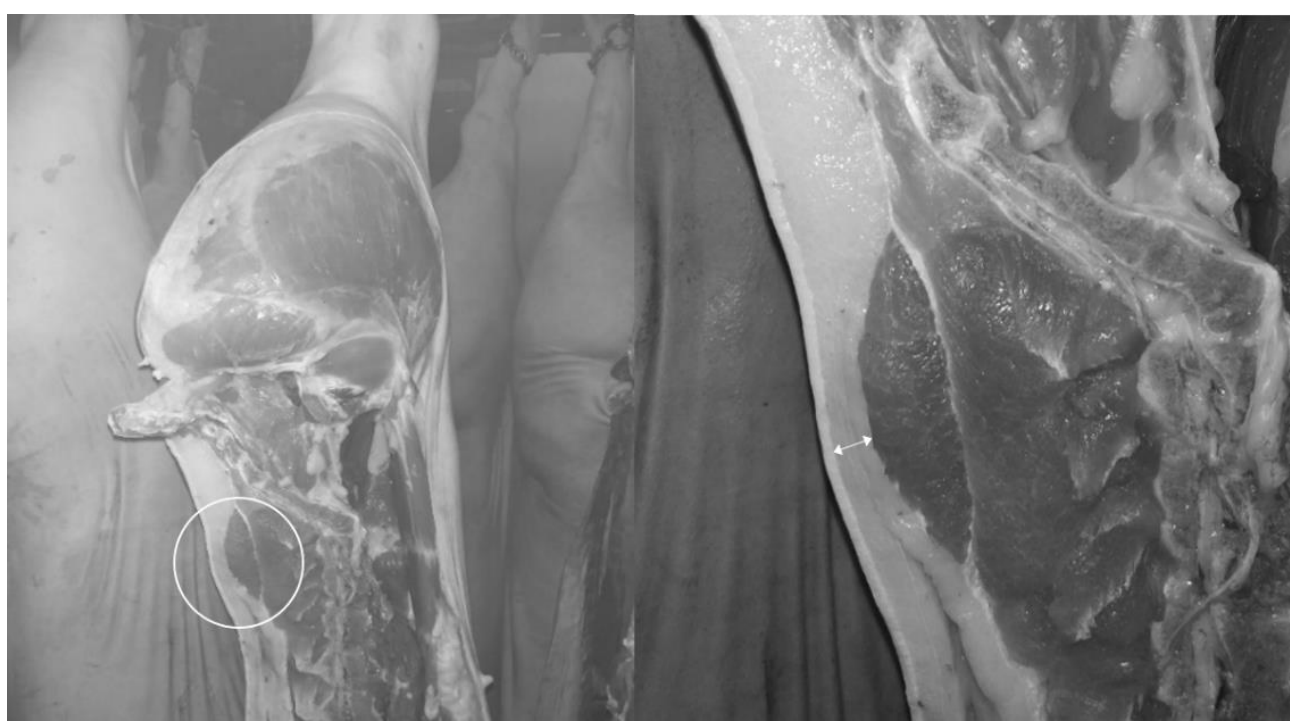

Fig. 1. Representation of the section used and the measure performed by an expert operator to measure the minimal fat thickness over muscle gluteus medius to obtain the classification target.

Table 2. Carcass classification according to minimal fat thickness over muscle gluteus medius based on a metrical measure with a ruler

\begin{tabular}{ll}
\hline Ham_Class $($ HC) & Fat depth $(\mathbf{m m})$ \\
\hline (1)- Thin & $<10$ \\
(2)- Standard & Between $<10$ and 15 \\
(3)- Semi-fat & Between $<15$ and 20 \\
(4)- Fat & $>20$ \\
\hline
\end{tabular}

\subsection{Dataset predictors}

AutoFom-III predicts carcass LMP and seven other variables (Table 3) from 48 parameters obtained from the scanning. Nevertheless, a more accurate handmade classification process of the ham is required for commercial purposes. With the aim of improving classification rates the eight estimations provided by AutoFom-III, that are going to be used as predictors, are complemented with three more predictors obtained in the production line (sex, breed, and weight) (Table 3). The extended set of 11 predictors was used as the input of automatic classification systems applying pattern recognition techniques to assess different classifiers.

Table 3. The eleven predictors used as the input of automatic classification systems

\begin{tabular}{lll}
\hline Predictor & Description & \\
& & Autofom III \\
\hline LMP & Lean Meat Percentage &
\end{tabular}


F34 According to the official formula, the subcutaneous fat thickness at $60 \mathrm{~mm}$ in the mid-line between the $3^{\text {rd }}$ and the $4^{\text {th }}$ last rib. $(\mathrm{mm})$

M34 According to the official formula, muscle thickness at $60 \mathrm{~mm}$ in the mid-line from the $3^{\text {rd }}$ to the $4^{\text {th }}$ last rib. $(\mathrm{mm})$

F_GM1 The minimum subcutaneous fat plus skin thickness measured with a ruler over the muscle

F GM2 The thickness of the subcutaneous fat plus skin measured with a ruler, perpendicularly to the

skin, at the cranial part of muscle Gluteus medius. (mm)

WGT_H Total weight of the ham $(\mathrm{kg})$

WGT_HWB Ham's weight without bone $(\mathrm{kg})$

WGT_HLM Total weight of the lean meat of the ham $(\mathrm{kg})$

\section{Production line}

\begin{tabular}{ll}
\hline SEX & Sex of animals (females, entire males and castrated males) \\
BREED & $\begin{array}{l}\text { Crossbreed ((Large White x Landrace) } x \text { Pietrain, , (Large White x Landrace) x Duroc, and } \\
\text { (Large White x Landrace) x (Duroc x Landrace)) }\end{array}$ \\
WGT & Cold carcass weight $(\mathrm{kg})$ \\
\hline
\end{tabular}

145

146 Finally, the HC parameter (1, 2, 3 or 4; see Table 2$)$ used as a response was scored by an expert operator and is referred to the manual metrical measure to classify hams according to the thickness of the fat at the point shown in Fig.1.

\subsection{Predictors and classifiers evaluated}

151 A preliminary study was performed to evaluate the potential of each predictor individually to 152 forecast the HC classification. Therefore, each single predictor was only considered to feed each 153 of the classifiers to obtain the response. All classifiers were evaluated in terms of the accuracy 154 which is defined as the number of correct predictions divided by the number of total predictions.

155 Moreover, the impact in the prediction of $\mathrm{HC}$ when taking different combinations of predictors 156 as inputs in the classifier was also assessed in terms of the accuracy. The aim of this assessment 157 was to compare the predictability of the classifiers when trained with only the single input LMP, 158 and when other predictors are incorporated, such as the combinations of LMP and SEX or LMP 159 and BREED (see Table 4) for all the combinations. These combinations were chosen according 160 to the different slaughterhouse scenarios described below.

\section{Predictors used as inputs}

\begin{tabular}{|c|c|c|c|c|c|c|c|c|c|c|c|}
\hline Datasets & $\overline{\text { LMP }^{1}}$ & $\overline{\text { SEX }^{2}}$ & WGT $^{3}$ & BREED $^{4}$ & $\mathrm{~F} 34^{5}$ & M346 & $\overline{\text { F_GM1 }}{ }^{7}$ & $\bar{F}$ GM2 $2^{8}$ & $\overline{\text { WGT_H }^{9}}$ & WGT_HWB $^{10}$ & WGT_HLM $^{11}$ \\
\hline D1 & $\mathrm{X}$ & & & & & & & & & & \\
\hline D2 & $\mathrm{X}$ & $X$ & & & & & & & & & \\
\hline D3 & $X$ & & $X$ & & & & & & & & \\
\hline D4 & $\mathrm{X}$ & & & $X$ & & & & & & & \\
\hline D5 & $X$ & $X$ & $X$ & $X$ & & & & & & & \\
\hline D6 & $X$ & $X$ & $X$ & $X$ & $X$ & $X$ & & & & & \\
\hline
\end{tabular}


${ }^{1}$ LMP (Lean Meat Percentage); ${ }^{2}$ SEX (females, entire males and castrated males); ${ }^{3} \mathrm{WGT}$ (warm carcass weight); ${ }^{4} \mathrm{BREED}$ ( (Large White $\mathrm{x}$ Landrace) x Pietrain, (Large White x Landrace) x Duroc and (Large White x Landrace) x (Duroc x Landrace)); ${ }^{5} \mathrm{~F} 34$ (subcutaneous fat thickness at $60 \mathrm{~mm}$ in the mid-line between the $3^{\text {rd }}$ and the $4^{\text {th }}$ last rib); ${ }^{6} \mathrm{M} 34$ (loin depth in mmmeasured at $60 \mathrm{~mm}$ from the midline between the $3^{\text {rd }}$ and the $4^{\text {th }}$ last rib); ${ }^{7}$ F_GM1 (minimum subcutaneous fat plus skin thickness measured with a ruler over the muscle Gluteus medius); ${ }^{8} \mathrm{~F}$ _GM2 (thickness of the subcutaneous fat plus skin measured with a ruler, perpendicularly to the skin, at the cranial part of muscle Gluteus medius); ${ }^{9}$ WGT_H (total weight of the ham); ${ }^{10}$ WGT_HWB (ham's weight without bone); ${ }^{11}$ WGT_HLM(total weight of the lean meat of the ham).

170

171

172

173

174

175

176

177

According to the Commission Delegated Regulation (EU) 2017/1182, it is mandatory in all the slaughterhouses to classify pig carcasses by means of its LMP. Therefore the combination D1 (Table 4) is available in the production line of all slaughterhouses.

As more procedures are added in the slaughtering line, more predictors could be obtained in realtime such as SEX, BREED, and WGT. Those additional predictors can be incorporated as inputs in the classifiers, as it has been done from D2 to D5.

Combination D6 considers the addition of predictors F34 and M34 that are provided by AutoFOM III. These predictors have been chosen because they can be assessed using other classification systems like Fat-O-Meat'er- FOM (Kempster, Chadwick, \& Jones, 1985). Finally, D7 takes all additional information given by AutoFOM III (predictors F_GM1, F_GM2, WGT_H, WGT_HWB, and WGT_HTL) (Table 4.).

\subsection{Statistical analysis}

To train each classifier four sets of 1000 samples of each HC class were randomly selected from the total of 31188 ones to form a balanced group of 4000 samples. Afterwards, to prevent the classifier overfitting, a 5-Fold cross-validation method was used (Bishop, 2006) dividing the dataset into 5 subsets, and for 5 times one of the 5 subsets was used as test set and the other 4 subsets get together to form a training set and the average error across all 5 trials was computed.

All classifiers were evaluated in terms of the accuracy (number of correct predictions divided by the number of total predictions).

A set of well-known classifier techniques was evaluated (Bishop, 2006): (1) Decision Trees: this type of algorithm is based on the construction of an automatic diagram of branches that appear according to the available data and the specific weight of each parameter. This algorithm was used with 4, 20 and 100 maximum split-levels; (2) Support Vector Machines (SVM): a discriminative classifier that separates classes by a hyperplane. The SVM algorithm is based on finding the optimal separating hyperplane that gives the largest minimum distance between the classes of the training data. This algorithm was used with four different kernels - linear, quadratic, 
cubic and Gaussian (Burges, 1998; Vapnik \& Chervonenkis, 1964); (3) K-Nearest Neighbour Classifiers $(K-\mathrm{NN})$ : a non-parametric supervised classifier based on the comparison of a sample against the $K$ samples which most resemble assigning the most abundant class (Cover \& Hart, 1967). This algorithm was used with six different configurations; (4) Discriminant Analysis with linear (Balakrishnama \& Ganapathiraju, 1998; Fisher, 1936) and quadratic configurations based on finding a linear or quadratic combination of parameters that characterise or separates two or more classes.

MATLAB and Signal Processing Toolbox ${ }^{\text {TM }}$ (Matlab R2016b; The MathWorks, Inc, 1988-2016) have been used to develop and test all the models and algorithms.

\section{Results and discussion}

Table 5 shows the accuracy of 17 classification models when a single predictor is taken as input. These classification models allow interpreting the results as a measure of the impact that each predictor by itself has in the forecast. Accuracy oscillates between 15 and 68\% depending on the predictor and the type of classifier. Predictors F_GM1 and F_GM2 obtain the best results of accuracy in most of the classifiers, outperforming the results obtained by LMP. F34 also achieves good results regarding accuracy, however, in this case, the results are more dependent on the classifier type. Those results were foreseeable as predictors F_GM1, F_GM2 and F34 provide information about a direct measure of fat thickness in two points of the ham and in one point of the loin, respectivily. Indeed, they are physically related to the handmade measure taken by an expert operator who assigns the HC class. On the other hand, predictors such as SEX, WGT and BREED can be good predictors to classify the hams correctly but largely depends on the type of classifier.

The highest and the lowest accuracy values for each predictors' dataset are presented in bold and underlined, respectively. The best results of predictors F_GM1, F34, F_GM2 and LMP predicted the $\mathrm{HC}$ class with an accuracy between 63 and 68\%. Moreover, predictors BREED, WGT and SEX predicted the HC class with an accuracy between 42 and $48 \%$. Finally, the rest of predictors, had an accuracy below $37 \%$.

In general, SVM Medium Gaussian or Coarse Gaussian or the Fine worked better when predictors are lean or fat parameters while SVM Cubic is one of the worst. This result persists in all predictors used but the interpretation about the relation of SVM kernels and the dataset is not clear.

When weight predictors are used, linear or quadratic discriminant analysis, and also Medium Gaussian, Coarse Gaussian and fine SVM produce the highest accuracy. These results suggest that continuous variables, such as the weight, improve the accuracy of more complex algorithms 
230 while categorical variables fits better with more simple algorithms. Sex and breed have higher

231 accuracy when decision trees and SVM are used and discriminant analysis for breed. We can

232 hypothesize than sex and breed obtain higher accuracy in decisions trees because, in the dataset,

233 they are only three breed classes (Table 1). According to the results of (Gispert et al 2007), there

234 is a clear relation between breed and SFT that could be easily formalized in simple decision trees.

235 Similar relations have been found for sex (Font-i-Furnols et al., 2012; Gispert et al., 2010). The

236 lowest accuracy is for the kNN approach. We can observe that for the classification of ham is

237 usually more relevant breed than weight, and in turn, weight than sex.

238 Table 5 The Accuracy (in percentage) to predict the Ham Classification (HC) based on the thickness of the 239 subcutaneous fat of the ham for each classifier when a single predictor is considered.

\begin{tabular}{|c|c|c|c|c|c|c|c|c|c|c|c|}
\hline \multirow[b]{2}{*}{ Classifiers } & \multicolumn{11}{|c|}{ Predictors } \\
\hline & $\mathbf{L M P}^{1}$ & F34 & M34 $^{6}$ & F_GM1 $^{7}$ & F_GM2 ${ }^{8}$ & WGT_H ${ }^{9}$ & WGT_HWB ${ }^{10}$ & WGT_HTL ${ }^{11}$ & SEX $^{2}$ & WGT $^{3}$ & BREED $^{4}$ \\
\hline \multicolumn{12}{|c|}{ Decision Trees } \\
\hline Simple tree & 62 & 65 & 36 & 68 & 63 & 33 & 33 & 32 & 42 & 44 & 48 \\
\hline Medium tree & 61 & 65 & 36 & 67 & 64 & 33 & 32 & 32 & 42 & 43 & 48 \\
\hline Complex tree & 61 & 63 & 36 & 65 & 62 & 32 & 32 & 29 & 42 & 42 & 48 \\
\hline \multicolumn{12}{|c|}{ Support Vector Machines } \\
\hline Linear & 52 & 58 & 27 & 59 & 51 & 28 & 27 & 26 & 40 & 35 & 47 \\
\hline Quadratic & 31 & 38 & 25 & 40 & 44 & 25 & 25 & 26 & 42 & 27 & 48 \\
\hline Cubic & $\underline{15}$ & $\underline{19}$ & $\underline{25}$ & 35 & $\underline{22}$ & $\underline{24}$ & $\underline{23}$ & $\underline{24}$ & 42 & $\underline{19}$ & 48 \\
\hline Fine & 63 & 65 & 36 & 68 & 65 & 34 & 33 & 32 & 42 & 44 & 48 \\
\hline Medium Gaussian & 63 & 65 & 36 & 68 & 65 & 35 & 33 & 32 & 42 & 44 & 48 \\
\hline Coarse Gaussian & 63 & 65 & 36 & 68 & 64 & 34 & 33 & 32 & 42 & 44 & 48 \\
\hline \multicolumn{12}{|c|}{ K-Nearest Neighbours } \\
\hline Fine & 36 & 53 & 30 & 57 & 53 & 27 & 28 & 25 & $\underline{25}$ & 26 & $\underline{25}$ \\
\hline Medium & 58 & 62 & 33 & 65 & 63 & 31 & 32 & 28 & $\underline{25}$ & 33 & $\underline{25}$ \\
\hline Coarse & 62 & 65 & 35 & 68 & 64 & 32 & 32 & 31 & 27 & 41 & $\underline{25}$ \\
\hline Cosine & 25 & 25 & $\underline{25}$ & $\underline{25}$ & 25 & 25 & 25 & 25 & $\underline{25}$ & 25 & $\underline{25}$ \\
\hline Cubic & 58 & 61 & 33 & 65 & 63 & 32 & 32 & 28 & $\underline{25}$ & 32 & $\underline{25}$ \\
\hline Weighted & 57 & 56 & 31 & 60 & 55 & 29 & 30 & 27 & 25 & 32 & 25 \\
\hline \multicolumn{12}{|c|}{ Discriminant analysis } \\
\hline Linear & 62 & 65 & 36 & 68 & 64 & 34 & 33 & 32 & 34 & 44 & 48 \\
\hline Quadratic & 61 & 64 & 36 & 68 & 63 & 35 & 34 & 32 & 39 & 44 & 48 \\
\hline
\end{tabular}



White x Landrace) x Pietrain, (Large White x Landrace) x Duroc and (Large White x Landrace) x (Duroc x Landrace)); ${ }^{5} \mathrm{~F} 34$ (subcutaneous fat thikness at $60 \mathrm{~mm}$ in the mid-line between the $3^{\text {rd }}$ and the $4^{\text {th }}$ last rib); ${ }^{6} \mathrm{M} 34$ (loin depth in mm measured at $60 \mathrm{~mm}$ from the midline between the $3^{\text {rd }}$ and the $4^{\text {th }}$ last rib); ${ }^{7}$ F_GM1 (minimum subcutaneous fat plus skin thickness measured with a ruler over the muscle Gluteus medius); ${ }^{8}$ F_GM2 (thickness of the subcutaneous fat plus skin measured with a ruler, perpendicularly to the skin, at the cranial part of muscle Gluteus medius); ${ }^{9}$ WGT_H (total weight of the ham); ${ }^{10}$ WGT_HWB (ham's weight without bone); ${ }^{11}$ WGT_HLM(total weight of the lean meat of the ham). In bold he highest value for each dataset; Underlined lowest value for each dataset.

249 Table 6 shows the accuracy of each classifier according to the data set configurations that are 250 more commonly available in different slaughterhouse scenarios, as described in section 2.3, Table

251 4. As commented in section 2.4 classifiers were obtained and validated with cross validation with 252 the 4000 carcasses. In addition, although the 27188 were a non-balanced data set in terms of HC, 253 (i.e. 16920 (thin), 6074 (standard), 4003 (semi-fat) and 191 (fat)) the classifiers were also 254 validated using this dataset and accuracy of the results was similar to the obtained by cross 255 validation (data not shown).

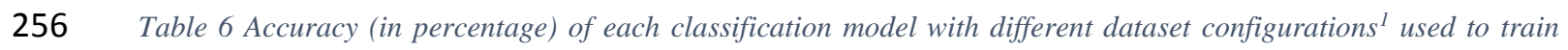
257 models.

\begin{tabular}{|c|c|c|c|c|c|c|c|}
\hline \multirow[b]{2}{*}{ Classifiers } & \multicolumn{7}{|c|}{ Datasets } \\
\hline & D1 & D2 & D3 & D4 & D5 & D6 & D7 \\
\hline \multicolumn{8}{|c|}{ Decision Trees } \\
\hline Simple tree & 62 & 62 & 63 & 62 & 64 & 65 & 68 \\
\hline Medium tree & 61 & 64 & 65 & 62 & 67 & 68 & 70 \\
\hline Complex tree & 61 & 63 & 64 & 61 & 68 & 67 & 68 \\
\hline \multicolumn{8}{|c|}{ Support Vector Machines } \\
\hline Linear & 52 & 61 & 67 & 63 & 69 & 71 & 71 \\
\hline Quadratic & 31 & 45 & 60 & 49 & 68 & 71 & 72 \\
\hline Cubic & $\underline{15}$ & $\underline{32}$ & $\underline{33}$ & $\underline{37}$ & 68 & 71 & 69 \\
\hline Fine & 63 & 65 & 66 & 63 & 68 & 69 & 69 \\
\hline Medium Gaussian & 63 & 65 & 67 & 63 & 69 & 70 & 73 \\
\hline Coarse Gaussian & 63 & 64 & 67 & 63 & 68 & 71 & 71 \\
\hline \multicolumn{8}{|c|}{ K-Nearest Neighbours } \\
\hline Fine & 36 & 43 & 56 & 42 & 59 & $\underline{61}$ & $\underline{62}$ \\
\hline Medium & 58 & 61 & 64 & 58 & 65 & 66 & 68 \\
\hline Coarse & 62 & 64 & 56 & 62 & 65 & 68 & 67 \\
\hline Cosine & 25 & 61 & 56 & 58 & 65 & 68 & 68 \\
\hline Cubic & 58 & 61 & 65 & 57 & 65 & 68 & 68 \\
\hline
\end{tabular}


$\begin{array}{llllllll}\text { Weighted } & 57 & 59 & 60 & \mathbf{6 3} & 63 & 67 & 68\end{array}$

Discriminant Analysis

\begin{tabular}{lccccccc}
\multicolumn{8}{c}{ Discriminant Analysis } \\
\hline Linear & 62 & 56 & 67 & 59 & 64 & 67 & 70 \\
Quadratic & 62 & 58 & 65 & 54 & $\underline{55}$ & 63 & 66 \\
\hline
\end{tabular}

258

259

260

261

262

263

264

265

266

267

268

269

270

271

272

273

274

275

276

277

278

279

280

281

282

283

284

285

286

287

In bold the highest value for each dataset; Underlined the lowest value for each dataset.

${ }^{1}$ See Table 2 for description of the inputs included as predictors in each dataset studied (from D1 to D7).

The first column shows the results obtained using LMP as a single predictor. The highest value (stood out in bold, Table 6) of the different classifiers for dataset configurations. D2 and D3 show a positive impact on most of the classifiers accuracy due to the incorporation of SEX and WGT predictors, respectively, compared with D1. Moreover, dataset configuration D4, in which BREED predictor has been incorporated, the accuracy improves just in some of the classifiers, such as SVM Linear and KNN Cosine. Predictor WGT seems to better complement LMP than SEX and BREED according to results obtained by Latorre, García-Belenguer, \& Ariño (2008).

As a general rule, SVM Coarse, SVM Medium Gaussian and SVM Fine obtain the highest accuracy when only one or two predictors are used (D1 to D4) compared with the other classifiers. Moreover, when more predictors are used, all the SVM classifiers produce better results than the other classifier techniques. In addition, the more predictors are added, the better results are obtained with the most sophisticated classifiers, such as SVMs with complex kernels.

When SEX, WGT and BREED predictors complement LMP (D5) the accuracy of SVM Medium Gaussian, one of the classifiers with the highest accuracy in D1, increases a 6\%, obtaining an accuracy value of 69\%. Furthermore, the SVM Linear with D5, also obtain an accuracy value of $69 \%$ increasing by $17 \%$ with respect to D1.

D6 dataset configuration incorporates to D5 predictors F34 and M34 obtained by Autofom. Configuration D7 has all available predictors (see section 2.2), obtained through the use of Autofom and intrinsic characteristics of the animal. In configurations D6 and D7, the classifiers obtain a percentage of accuracy between 61 and 73\%. As expected, D7 configuration obtains the best performance. Regarding the classifiers, the SVM Medium Gaussian reached the best result with a percentage of accuracy of $73 \%$.

When comparing models obtained from datasets D6 and D7, in average, there is a $1.0 \%$ of prediction improvement. It is suggested that the improvement is not greater because the added parameters are closely correlated with the previous ones. For instance, the five new predictors (F_GM1, FGM2, WGT_H, WGT_HWB, WGT_HLM) introduced in the models with input dataset D7 are highly correlated with predictors WGT and/or F34, present in dataset D6. However, although an increase of $1.0 \%$ does not represent a great improvement in terms of percentage of 

of a ham in a lower category, in terms of subcutaneous fat, could incur in losses of more than $30 \%$ in the final sale price.

291

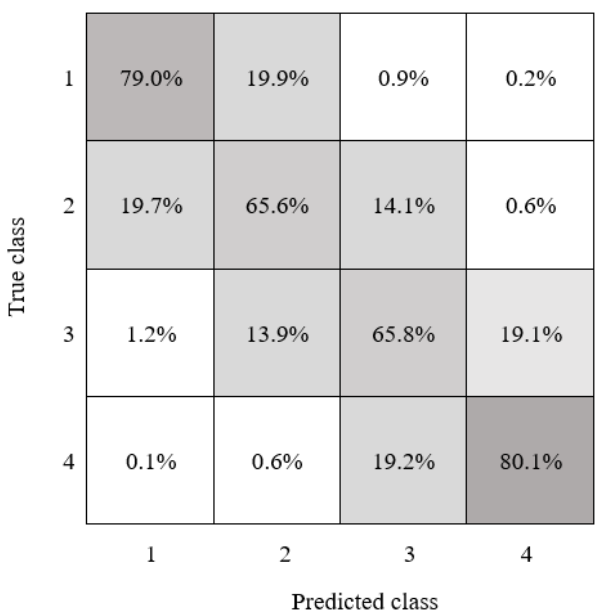

Fig. 2 Confusion Matrix of the best accuracy models obtained using SVM medium Gaussian model trained with all data available (D7). The results are given in percentage.

Fig.2 shows the confusion matrix obtained by SVM Medium Gaussian model developed using D7. The accuracy of HC classes 1 (79.0\%) and 4 (80.1\%) are higher than the accuracy of HC classes $2(65.6 \%)$ and $3(65.8 \%)$. When classes based on a metric threshold are used, extreme classes tend to be better classified.

The percentage of samples that are incorrectly classified into one of the adjacent categories varies between $13.9 \%-19.9 \%$ (Fig. 2.). It should be noted that some of these samples fall very close to the decision thresholds and, in those cases, the classification is particularly difficult.

Moreover, only less than $3.6 \%$ of the samples are misclassified in not adjacent categories. Indeed, it can be concluded that $96.7 \%$ of the $27.4 \%$ of misclassified samples correspond to samples classified into adjacent categories.

As explained before, all the models are developed in order to predict the classification of the hams by an expert operator. Indeed, in this study the human classification methodology is used as "golden standard" despite the fact that this methodology presents some difficulties such as operator fatigue (Font-i-Furnols et al., 2016; Olsen et al., 2007) but also the evaluation of the fat thickness after the carcass being split down by an industrial robot (the carcasses are not precisely split down in the same way). Therefore, misclassifications of the models do not always mean that the model is classifying wrong, they are just explaining that the model classification does not match with the human classification.

Nowadays, the SVM Medium Gaussian model is applied in MAFRICA S.A. slaughterhouse. It is observed an accuracy improvement which is not currently quantified. Our working hypothesis 
314 is that automatic classification improves manual classification because decision making is

315 objective and operator fatigue are eliminated.

316 4. Conclusions

317 Pattern recognition models, based on data usually available on slaughterhouses, can be used to

318 classify the hams according to the thickness of the subcutaneous fat, and this classification can

319 emulate the manual system with an effectivity of $73 \%$. This result suggests that pattern

320 recognition models can be a useful online tool to increase slaughterhouses' benefits because more

321 accurate classification increases optimization of the ham processing.

\section{Acknowledgements}

"Financial support from the Agency for Management of University and Research Grants (AGAUR) of the Catalan Government to Gerard Masferrer is grateful". CERCA Programme (Generalitat de Catalunya) is also acknowledged.

\section{References}

Andersen, H. (n.d.). AutoFom III TM -using data for genetic development and payment. Retrieved from http://www.carometec.com/images/zoo/pdf/brochures/ApplicationNote_English.pdf

Balakrishnama, S., \& Ganapathiraju, A. (1998). Linear Discriminant Analysis - a Brief Tutorial. Compute, 11, 1-9. http://doi.org/http://www.isip.piconepress.com/publications/reports/1998/isip/lda/

Bishop, C. M. (2006). Pattern Recognition and Machine Learning. Pattern Recognition (Vol. 4). http://doi.org/10.1117/1.2819119

Bosi, P., Russo, V., \& Paolo, B. (2004). The production of the heavy pig for high quality processed products. J.ANIM.SCI, 3(21), 309-32145. http://doi.org/10.4081/ijas.2004.309

Burges, C. J. C. (1998). A Tutorial on Support Vector Machines for Pattern Recognition. Data Mining and Knowledge Discovery, 2(2), 121-167. http://doi.org/10.1023/A:1009715923555

Candek-Potokar, M., \& Skrlep, M. (2012). Factors in pig production that impact the quality of dry-cured ham: a review. Animal : An International Journal of Animal Bioscience, 6(2), 327-38. http://doi.org/10.1017/S1751731111001625

Carabús, A., Gispert, M., Brun, A., Rodríguez, P., \& Font-i-Furnols, M. (2014). In vivo computed tomography evaluation of the composition of the carcass and main cuts of 
growing pigs of three commercial crossbreeds. Livestock Science, 170, 181-192. http://doi.org/10.1016/j.livsci.2014.10.005

Cilla, I., Altarriba, J., Guerrero, L., Gispert, M., Martínez, L., Moreno, C., ... Roncalés, P. (2006). Effect of different Duroc line sires on carcass composition, meat quality and drycured ham acceptability. Meat Science, 72(2), 252-260. http://doi.org/10.1016/j.meatsci.2005.07.010

Cisneros, F., Ellis, M., \& McKeith, F. (1996). Influence of slaughter weight on growth and carcass characteristics, commercial cutting and curing yields, and meat quality of barrows and gilts from two genotypes. Journal of Animal. Retrieved from https://dl.sciencesocieties.org/publications/jas/abstracts/74/5/925

Commission Delegated Regulation (EU) 2017/1182 of 20 April 2017 supplementing Regulation (EU) No 1308/2013 of the European Parliament and of the Council as regards the Union scales for the classification of beef, pig and sheep carcasses and as regards the reporting of market prices of certain categories of carcasses and live animals. Official Journal of the European Union 4.7.17, L171/74-L171-99.

Cover, T., \& Hart, P. (1967). Nearest neighbor pattern classification. IEEE Transactions on Information Theory, 13(1), 21-27. http://doi.org/10.1109/TIT.1967.1053964

Daumas, G. (2011). Non-electronic techniques to classify pig carcasses in small slaughterhouses. In Second International Virtual Conference on pork Quality. Novembere, 05to Decembere (p. p 06-2001). Retrieved from https://www.researchgate.net/profile/Gerard_Daumas/publication/285574394_NONELECTRONIC_TECHNIQUES_TO_CLASSIFY_PIG_CARCASSES_IN_SMALL_SLA UGHTERHOUSES/links/565efc6308ae1ef92984212f.pdf

Dirinck, P., Van Opstaele, F., \& Vandendriessche, F. (1997). Flavour differences between northern and southern European cured hams. Food Chemistry, 59(4), 511-521. http://doi.org/10.1016/S0308-8146(97)00012-5

Edwards, D. B., Bates, R. O., \& Osburn, W. N. (2003). Evaluation of Duroc- vs. Pietrain-sired pigs for carcass and meat quality measures. Journal of Animal Science, 81(8), 1895. http://doi.org/10.2527/2003.8181895x

Fàbrega, E., Gispert, M., Tibau, J., Hortós, M., Oliver, M. A., \& Font i Furnols, M. (2011). Effect of housing system, slaughter weight and slaughter strategy on carcass and meat quality, sex organ development and androstenone and skatole levels in Duroc finished entire male pigs. Meat Science, 89(4), 434-439. 
http://doi.org/10.1016/j.meatsci.2011.05.009

Fisher, R. A. (1936). The use of multiple measurements in taxanomic problems. Annals of Eugenics, 7(2), 179-188. http://doi.org/10.1111/j.1469-1809.1936.tb02137.x

Font-i-Furnols, M., Čandek-Potokar, M., Daumas, G., Gispert, M., Judas, M., \& Seynaeve, M. (2016). Comparison of national ZP equations for lean meat percentage assessment in SEUROP pig classification. Meat Science, 113, 1-8. http://doi.org/10.1016/j.meatsci.2015.11.004

Font-i-Furnols, M., Gispert, M., Soler, J., Diaz, M., Garcia-Regueiro, J. A., Diaz, I., \& Pearce, M. C. (2012). Effect of vaccination against gonadotrophin-releasing factor on growth performance, carcass, meat and fat quality of male Duroc pigs for dry-cured ham production. Meat Science, 91(2), 148-154. http://doi.org/10.1016/j.meatsci.2012.01.008

Font i Furnols, M., \& Gispert, M. (2009). Comparison of different devices for predicting the lean meat percentage of pig carcasses. Meat Science, 83(3), 443-446. http://doi.org/10.1016/j.meatsci.2009.06.018

Gispert, M., Àngels Oliver, M., Velarde, A., Suarez, P., Pérez, J., \& Font i Furnols, M. (2010). Carcass and meat quality characteristics of immunocastrated male, surgically castrated male, entire male and female pigs. Meat Science, 85(4), 664-670. http://doi.org/10.1016/j.meatsci.2010.03.021

Gispert, M., Font i Furnols, M., Gil, M., Velarde, A., Diestre, A., Carrión, D., ... Plastow, G. S. (2007). Relationships between carcass quality parameters and genetic types. Meat Science, 77(3), 397-404. http://doi.org/10.1016/j.meatsci.2007.04.006

Kempster, A. J., Chadwick, J. P., \& Jones, D. W. (1985). An evaluation of the Hennessy grading probe and the SFK Fat-O-Meater for use in pig carcass classification and grading. Animal Production, 40(2), 323-329. http://doi.org/10.1017/S0003356100025447

Latorre, M. A., García-Belenguer, E., \& Ariño, L. (2008). The effects of sex and slaughter weight on growth performance and carcass traits of pigs intended for dry-cured ham from Teruel (Spain). Journal of Animal Science, 86(8), 1933-1942. http://doi.org/10.2527/jas.2007-0764

Lucas, D., Brun, A., Gispert, M., Carabús, A., Soler, J., Tibau, J., \& Font-i-Furnols, M. (2017). Relationship between pig carcass characteristics measured in live pigs or carcasses with Piglog, Fat-o-Meat'er and computed tomography. Livestock Science, 197, 88-95. http://doi.org/10.1016/j.livsci.2017.01.010

Nanni Costa, L., Stelletta, C., Cannizzo, C., Gianesella, M., Lo Fiego, D. P., \& Morgante, M. 
(2010). The use of thermography on the slaughter-line for the assessment of pork and raw ham quality. Italian Journal of Animal Science, 6(1s). http://doi.org/10.4081/ijas.2007.1s.704

Olsen, E. V., Candek-Potokar, M., Oksama, M., Kien, S., Lisiak, D., \& Busk, H. (2007). Online measurements in pig carcass classification: Repeatability and variation caused by the operator and the copy of instrument. Meat Science, 75(1), 29-38. http://doi.org/10.1016/j.meatsci.2006.06.011

Realini, C. E., Duran-Montgé, P., Lizardo, R., Gispert, M., Oliver, M. A., \& Esteve-Garcia, E. (2010). Effect of source of dietary fat on pig performance, carcass characteristics and carcass fat content, distribution and fatty acid composition. Meat Science, 85(4), 606-612. http://doi.org/10.1016/j.meatsci.2010.03.011

Tous, N., Lizardo, R., Vilà, B., Gispert, M., Font-I-Furnols, M., \& Esteve-Garcia, E. (2014). Effect of reducing dietary protein and lysine on growth performance, carcass characteristics, intramuscular fat, and fatty acid profile of finishing barrows 1. J. Anim. Sci, 92, 129-140. http://doi.org/10.2527/jas2012-6222

Vapnik, V. N., \& Chervonenkis, A. (1964). A note on one class of perceptrons. Automation and Remote Control, 25(6), 937-945. Retrieved from https://scholar.google.es/scholar?q=Vladimir+Vapnik+Alexey+Ya.+Chervonenkis\&hl=ca \&as_sdt=0\%2C5\&as_ylo=1960\&as_yhi $=1965$

Wood, J. D., Enser, M., Fisher, A. V., Nute, G. R., Sheard, P. R., Richardson, R. I., ... Whittington, F. M. (2008). Fat deposition, fatty acid composition and meat quality: A review. Meat Science. http://doi.org/10.1016/j.meatsci.2007.07.019

Wood, J. D., Enser, M., Whittington, F. M., Moncrieff, C. B., \& Kempster, A. J. (1989). Wood et al., 1989 Backfat composition in pigs Differences between fat thickness groups and sexes..pdf. Livestock Production Science Elsevier Science Publishers B.V. Retrieved from http://ac.els-cdn.com/0301622689900663/1-s2.0-0301622689900663main.pdf?_tid=02bf6b6e-300f-11e7-a6c900000aacb35d\&acdnat=1493822848_b5a764336449f25b0d2b04164c239dad

Wood, J. D., Nute, G. R., Richardson, R. I., Whittington, F. M., Southwood, O., Plastow, G., ... Chang, K. C. (2004). Effects of breed, diet and muscle on fat deposition and eating quality in pigs. Meat Science, 67(4), 651-667. http://doi.org/10.1016/j.meatsci.2004.01.007 